\title{
Rigidly Foldable Origami Twists
}

Thomas A. Evans

Brigham Young University - Provo

Robert J. Lang

Lang Origami

Spencer P. Magleby

Brigham Young University - Provo

Larry L. Howell

Brigham Young University - Utah, Ihowell@byu.edu

Follow this and additional works at: https://scholarsarchive.byu.edu/facpub

Part of the Mechanical Engineering Commons

\section{Original Publication Citation}

Thomas A. Evans, Robert J. Lang, Spencer P. Magleby, Larry L. Howell. Rigidly foldable origami gadgets and tessellations. R. Soc. open sci. 20152 150067; DOI: 10.1098/rsos.150067.

Published 16 September 2015,

\section{BYU ScholarsArchive Citation}

Evans, Thomas A.; Lang, Robert J.; Magleby, Spencer P.; and Howell, Larry L., "Rigidly Foldable Origami Twists" (2015). Faculty Publications. 1606.

https://scholarsarchive.byu.edu/facpub/1606

This Book Chapter is brought to you for free and open access by BYU ScholarsArchive. It has been accepted for inclusion in Faculty Publications by an authorized administrator of BYU ScholarsArchive. For more information, please contact ellen_amatangelo@byu.edu. 


\title{
Pre-publication version of: \\ Evans, T.A., Lang, R.J., Magleby, S.P., Howell, L.L., \\ "Rigidly Foldable Origami Twists," Origami 6, American \\ Mathematical Society, Vol. 1, pp. 119-130, 2015.
}

\section{RIGIDLY FOLDABLE ORIGAMI TWISTS}

\author{
THOMAS A. EVANS, ROBERT J. LANG, SPENCER P. MAGLEBY, \\ AND LARRY L. HOWELL
}

\section{INTRODUCTION}

Rigid foldability is an important characteristic of origami structures that becomes significant with non-paper materials. A rigidly foldable origami tessellation is one where the sectors remain rigid and all deflection occurs at the crease lines. Many rigidly foldable patterns have only one degree of freedom, making them potentially useful for deployable structures. Methods have been developed to construct rigidly foldable origami tessellations using materials with finite thickness based on zero-thickness rigidly foldable patterns. [Tachi 11].

Origami methods have been considered for application in deployable structures such as solar panels [Miura 85] [Zirbel et al. 13] and sterile shrouds [Francis et al. 13]. Other recent developments have included self-deployable origami stent grafts [Kuribayashi et al. 06], self-folding membranes [Pickett 07], and sandwich panel cores [Lebee and Sab 10]. A better understanding of how to create rigidly foldable tessellations can lead to previously unexplored applications.

This paper develops a method for evaluating the rigid foldability of origami tessellations by examining relationships between the dihedral angles in the pattern. The method is then used to determine what configurations allow origami twists, in particular, to be rigidly foldable. Rigidly foldable twists may be arrayed in a tessellation, providing a foundation for deployable origami-based structures to be constructed out of rigid materials.

\section{Rigidly Foldable Origami}

We will focus on patterns composed of degree- 4 vertices, where a typical vertex is illustrated in Figure 1. Four creases meet at each vertex; the paper between adjacent creases is a sector and the angle between adjacent creases is a sector angle, designated $\alpha$. The angle of the fold itself is the dihedral angle, denoted by $\gamma$, which is the angle between the surface normals of the two incident sectors. A crease may be a mountain fold $(\gamma<0)$, a valley fold $(\gamma>0)$, or unfolded $(\gamma=0)$. We will indicate valley folds by dashed lines and mountain folds by solid lines. We index the sector angles $\alpha_{i}$ and dihedral angles $\gamma_{i}$ so that sector $\alpha_{i}$ lies between folds $\gamma_{i}$ and $\gamma_{i+1}$, as illustrated in the figure.

2.1. Flat Foldability in Degree-4 Vertices. A flat-foldable vertex can be folded so that all dihedral angles are equal to $\pm \pi$. Likewise, an origami pattern is considered flat foldable if there exists a configuration where all dihedral angles in the pattern are equal to $\pm \pi$. The conditions for flat-foldability are well known (see, e.g., [Hull 03]); for degree-4 vertices, they can be summarized as:

- Opposite sector angles sum to $\pi$; 


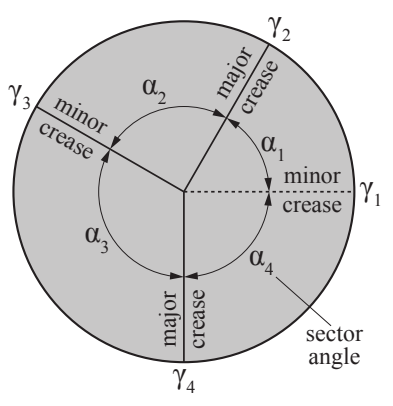

(a) Unfolded

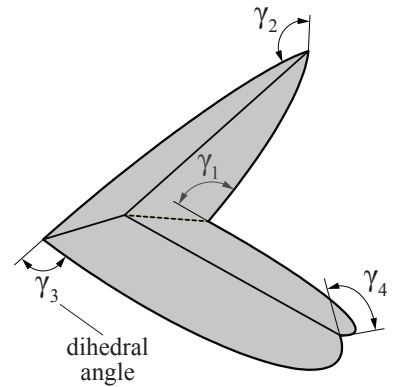

(b) Partially folded

Figure 1. A Degree-4 origami vertex is its (a) unfolded and (b) partially folded states.

- There exist three folds of one parity and one fold of the other;

- The smallest-angled sector, if unique, is incident to folds of opposite parity ("anto");

- The largest-angled sector, if unique, is incident to folds of the same parity ("iso").

If there exist two equal smallest angled sectors, at least one of these sectors must be anto and its opposite sector must be iso. We call the two opposite creases with equal parity the major creases and the other two the minor creases.

These conditions imply a relationship between the sector angles:

$$
\alpha_{1}+\alpha_{3}=\alpha_{2}+\alpha_{4}=\pi \text {. }
$$

These are necessary conditions, not sufficient; an origami pattern composed entirely from flat foldable vertices may still not be flat-foldable due to self-intersection. However, any origami tessellation containing one or more non-flat-foldable vertices cannot be flat-foldable.

2.2. Fold-Angle Multipliers. We now introduce relationships between dihedral angles in a flat-foldable degree-4 vertex. Huffman [Huffman 76], Lang [Hull 03], and Tachi [Tachi 10] derived several relationships (which were equivalent under trigonometric transformation). We present equivalent, but new, and somewhat simpler expressions here.

For the degree- 4 vertex of Figure 1 where $\gamma_{2,4}$ are the major creases and $\gamma_{1,3}$ are the minor creases, in all configurations between the unfolded and fully folded states, the following relationships apply:

$$
\gamma_{3}=-\gamma_{1}, \gamma_{2}=\gamma_{4}=2 \arctan \left(\frac{\sin \left(\frac{1}{2}\left(\alpha_{1}+\alpha_{2}\right)\right)}{\sin \left(\frac{1}{2}\left(\alpha_{1}-\alpha_{2}\right)\right)} \tan \left(\frac{1}{2} \gamma_{1}\right)\right)
$$

or equivalently,

$$
\frac{\tan \left(\frac{1}{2} \gamma_{2}\right)}{\tan \left(\frac{1}{2} \gamma_{1}\right)}=\frac{\tan \left(\frac{1}{2} \gamma_{4}\right)}{\tan \left(\frac{1}{2} \gamma_{1}\right)}=\frac{\sin \left(\frac{1}{2}\left(\alpha_{1}+\alpha_{2}\right)\right)}{\sin \left(\frac{1}{2}\left(\alpha_{1}-\alpha_{2}\right)\right)}
$$

The ratio between the half-angle tangents of any two dihedral angles in a flatfoldable degree- 4 vertex is a constant that depends solely on the (fixed) values of 


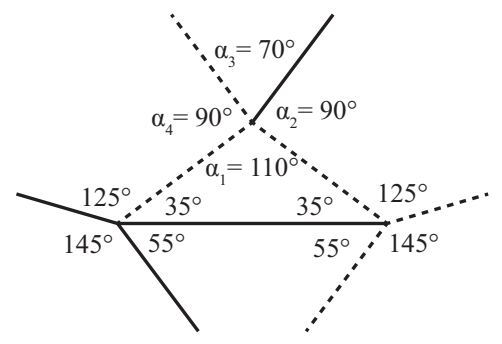

(a) Sector Angles

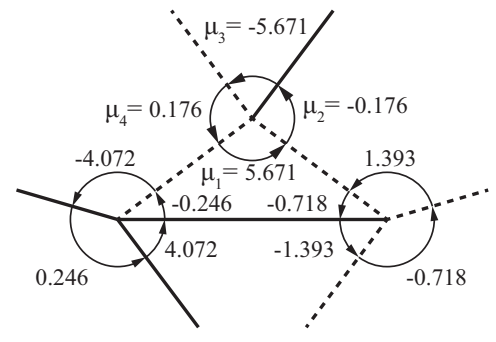

(b) Fold-Angle Multipliers

Figure 2. Rigidly foldable triangle. (a) Sector angles shown. (b) Fold angle multipliers for each consecutive pair of creases.

the sector anges. We call this ratio the "fold-angle multiplier", $\mu$ :

$$
\mu \equiv \frac{\sin \left(\frac{1}{2}\left(\alpha_{1}+\alpha_{2}\right)\right)}{\sin \left(\frac{1}{2}\left(\alpha_{1}-\alpha_{2}\right)\right)}=\frac{\tan \left(\frac{1}{2} \gamma_{2}\right)}{\tan \left(\frac{1}{2} \gamma_{1}\right)}=\frac{\tan \left(\frac{1}{2} \gamma_{4}\right)}{\tan \left(\frac{1}{2} \gamma_{1}\right)}
$$

We further define $\mu_{i}$ to be the ratio between the half-angle tangents of the dihedral angles adjacent to the $i^{t h}$ sector, i.e., $\mu_{i} \equiv \tan \left(\frac{1}{2} \gamma_{i+1}\right) / \tan \left(\frac{1}{2} \gamma_{i}\right)$. Then

$$
\mu_{1}=-\mu_{3}=\mu, \mu_{2}=-\mu_{4}=-\frac{1}{\mu}
$$

There is a special case to note: when the major crease fold lines are collinear $\left(\alpha_{2}+\alpha_{3}=\pi\right)$, zero and infinite fold-angle multipliers are obtained. This occurs because the major crease lines must be completely folded before the minor crease lines begin folding.

2.3. Rigidly Foldable Polygons. The fold angle multipliers $\left(\mu_{i}\right)$ capture the relationship between consecutive folds around a vertex. They can therefore be used to evaluate the rigid foldability of arrays of vertices. For an origami tessellation to be rigidly foldable, each vertex and each closed polygon in the tessellation must be rigidly foldable. (Again, there are longer-range self-intersection issues that must be considered for global rigid foldability, which we are intentionally not addressing.) For an $n$-degree polygon with interior angles 1 through $n$ the fold angle multipliers $\left(\mu_{i}\right)$ associated with the crease pairs at each vertex define a loop condition that enforces consistency around the polygon, namely

$$
\prod_{i=1}^{n} \mu_{i}=1
$$

Figure 2 shows the sector angles and fold-angle multipliers for a rigidly foldable triangle. Each vertex is rigidly foldable in isolation; since the product of the fold angle multipliers around the interior polygon is $1(-0.246 \times 5.671 \times-0.718=1)$, the entire pattern is similarly rigidly foldable.

\section{Rigid Foldability of Origami Twists}

The origami twist is a building block of many origami patterns that have application to deployable structures. It consists of a central polygon plus parallel pairs of creases extended from each side of the central polygon, as shown in Figure 3(a). 


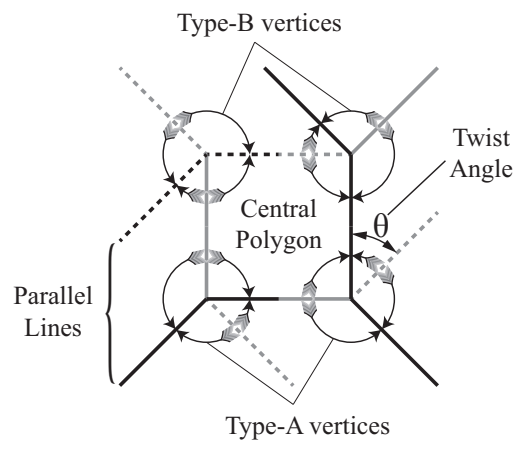

(a) Square twist

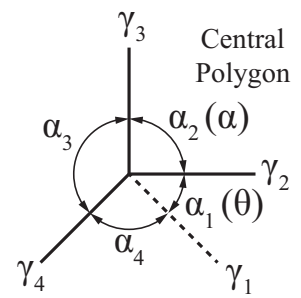

(b) Type-A Vertex

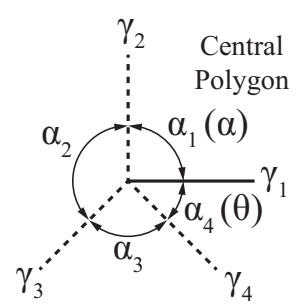

(c) Type-B Vertex

Figure 3. (a) A rigidly foldable square twist with twist angle $\theta$. Arrows point from the minor to the major crease lines. (b) A Type-A vertex. (c) A Type-B vertex.

The angle between each parallel pair and its adjacent side is the twist angle; in a flat-foldable origami twist, all vertices have the same twist angle.

We will classify twist vertices as one of two types. If conventional numbering is used and the first sector lies counterclockwise from a minor crease, a vertex is of Type-A if the sector which includes the central polygon is evenly numbered (see Figure 3(b)). Conversely, if this sector is oddly numbered, the vertex is of TypeB (see Figure 3(c)). Another method of differentiating vertex types is shown in Figure 3(a). If an arrow is drawn from minor crease lines to major crease lines at each vertex, a Type-A vertex will have a clockwise arrow in the central polygon while a Type-B vertex will have a counterclockwise arrow. A twist polygon can be characterized by the sequence of its vertices; the square twist of Figure 3(a) is AABB.

Note that zero and infinite multipliers can occur if the twist angle is equal to the interior angle of a Type-B vertex or if the twist angle is equal to the complement of the interior angle of a Type-A vertex.

For a Type-A vertex of a twist with interior angle $\alpha=\alpha_{2}$ and twist angle $\theta=\alpha_{1}$, we can evaluate the fold-angle multiplier $\left(\mu_{A}\right)$ for that vertex:

$$
\mu_{A}=-\frac{\sin \left(\frac{1}{2}(\theta-\alpha)\right)}{\sin \left(\frac{1}{2}(\theta+\alpha)\right)}=\frac{\sin \left(\frac{1}{2}(\alpha-\theta)\right)}{\sin \left(\frac{1}{2}(\alpha+\theta)\right)} .
$$

For a Type-B vertex with interior angle $\alpha=\alpha_{1}$ and twist angle $\theta=\alpha_{4}$, we have

$$
\mu_{B}=\frac{\sin \left(\frac{1}{2}(\alpha+\pi-\theta)\right)}{\sin \left(\frac{1}{2}(\alpha-\pi+\theta)\right)}=-\frac{\cos \left(\frac{1}{2}(\alpha-\theta)\right)}{\cos \left(\frac{1}{2}(\alpha+\theta)\right)} .
$$

With the constraints $0<\alpha<180^{\circ}$ and $0<\theta<180^{\circ}$, it follows that

$$
\left|\mu_{A}\right|<1<\left|\mu_{B}\right| \text {. }
$$

This gives us the following result.

Theorem 3.1 (No Rigidly Foldable Vertex-Uniform Twists). No origami twist with degree-4 vertices having all Type- $A$ or all Type-B vertices is rigidly foldable.

The proof follows directly from Equations 9 and 6. 


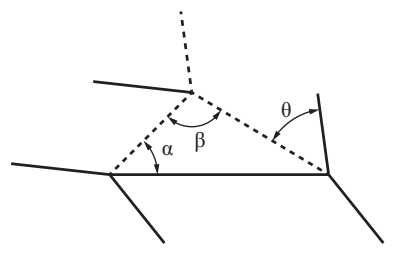

(a) $\mathrm{AAB}$

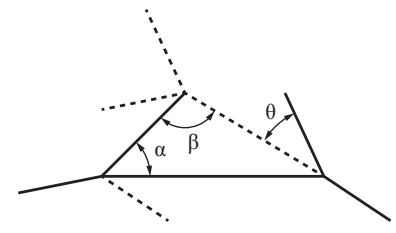

(b) $\mathrm{ABB}$

FiguRE 4. Two flat-foldable triangle twist configurations. The $\alpha$ and $\beta$ vertices in (a) are both Type-A while the third is Type- $\mathrm{B}$. The $\alpha$ vertex in (b) is Type-A while the other two are Type-B.

\section{Triangle Twists}

We now consider triangular twists. In this section we will prove the following:

Theorem 4.1 (No Rigidly Foldable Triangle Twist). No origami triangle twist is rigidly foldable.

To prove this theorem we will consider the two basic configurations with potential to be rigidly foldable for a triangle twist. These two configurations are ABA and $\mathrm{ABB}$ (cyclic permutations are equivalent).

For the ABA configuration (see Figure 4(a)), substituting Equations (7) and (8) into Equation (6) results in the condition

$$
\frac{\sin \left(\frac{\alpha-\theta}{2}\right) \sin \left(\frac{\beta-\theta}{2}\right) \cos \left(\frac{\alpha+\beta+\theta}{2}\right)}{\sin \left(\frac{\alpha+\theta}{2}\right) \sin \left(\frac{\beta+\theta}{2}\right) \cos \left(\frac{\alpha+\beta-\theta}{2}\right)}=1 .
$$

This equation is satisfied only if $\theta=\pi$ or $\alpha+\beta=2 \pi$. In the first case the angle opposite of the twist angle becomes zero and in the second case the triangle violates geometric compatibility. Therefore, there is no rigidly foldable ABA triangle twist.

For the ABB configuration (see Figure 4(b)), Equation (6) results in

$$
\frac{\sin \left(\frac{\alpha-\theta}{2}\right) \cos \left(\frac{\theta-\beta}{2}\right) \cos \left(\frac{\alpha+\beta+\theta}{2}\right)}{\sin \left(\frac{\alpha+\theta}{2}\right) \cos \left(\frac{\beta+\theta}{2}\right) \cos \left(\frac{\alpha+\beta-\theta}{2}\right)}=1
$$

This equation is satisfied only if $\theta=0$ or $\alpha+\beta=\pi$. In the first case the twist angle becomes zero and in the second case the third interior angle becomes zero. Therefore, there is no rigidly foldable ABB triangle twist. Because all other triangle twists with two Type-A and one Type-B or two Type-B and one Type-A vertices may be obtained by rotating the $\mathrm{AAB}$ or $\mathrm{ABB}$ twists, we conclude that no triangle twist is rigidly foldable. Because our definition of an origami twist requires parallel pleats, Theorem 4.1 does not eliminate the possibility of rigidly foldable triangles with non-parallel pleats.

\section{Quadrilateral Twists}

For simplification, quadrilateral twists have been divided into several standard types of quadrilaterals. These are discussed below. 


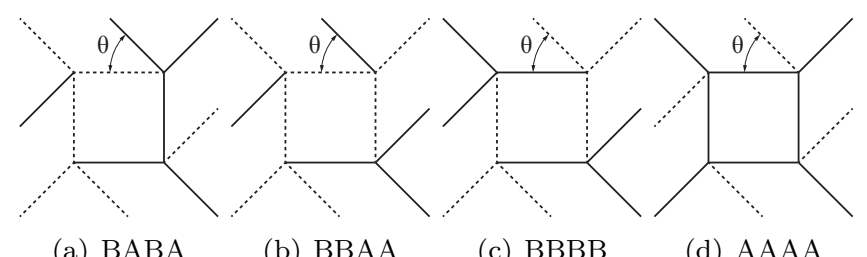

Figure 5. Flat foldable rectangular twists. (a) and (b) are rigidly foldable while (c) and (d) are not. The vertex label for each twist starts at the lower left vertex and runs counterclockwise.

5.1. Rectangle and Square Twists. Since side length does not factor into the fold-angle multipliers, square and rectangular twists have the same conditions for rigid foldability.

Theorem 5.1 (Rectangle and Square Twists). A square or rectangular twist is rigidly foldable if and only if it contains two Type- $A$ and two Type-B vertices, with a twist angle not equal to $90^{\circ}$.

The proof is as follows. For a rectangular twist, $\alpha=90^{\circ}$. Therefore, Equations (7) and (8) simplify to give the following result:

$$
\begin{gathered}
\mu_{A}=\cot \left(\frac{\pi}{4}+\frac{\theta}{2}\right) \\
\mu_{B}=-\tan \left(\frac{\pi}{4}+\frac{\theta}{2}\right)
\end{gathered}
$$

As can be seen from Equations (12) and (13), the fold angle multipliers for TypeA and Type-B vertices in such a twist are negative reciprocals of one another. Therefore, any rectangular twist with two Type-A and two Type-B vertices will satisfy Equation (6) and is rigidly foldable. The only exception is when $\theta=90^{\circ}$, where multipliers become infinite. Conversely, those with unequal numbers of TypeA and Type-B vertices cannot satisfy Equation (6) and are not rigidly foldable. Figure 5 shows all of the possibilities (to within cyclic permutation and/or global parity reversal).

5.2. Parallelogram, Rhombus, and Isosceles Trapezoid Twists. All three of these types of polygons contain two sets of supplementary interior angles, although the order in which these angles are arranged differs. Since this order does not affect rigid foldability, rhombus, parallelogram, and isosceles trapezoid twists have the same conditions for rigid foldability.

Theorem 5.2 (Parallelogram, Rhombus, and Isosceles Trapezoid Twists). A parallelogram, rhombus, or isosceles trapezoid twist is rigidly foldable if and only if it contains two Type- $A$ and two Type- $B$ vertices, with a twist angle not equal to the interior angle of a Type- $A$ vertex.

To prove this we will define $\alpha$ as the value of one of the interior angles of the polygon. Of necessity, there are two interior angles with a value of $\alpha$ and two with a value of $\pi-\alpha$. The multipliers for the $\alpha$-vertices may be calculated using Equations 


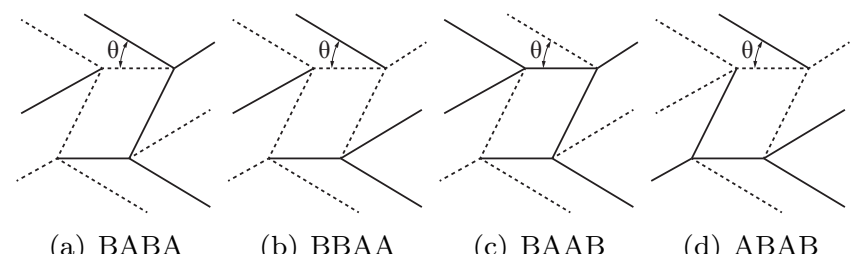
(a) BABA
(b) BBAA
(c) $\mathrm{BAAB}$
(d) $\mathrm{ABAB}$

FiguRE 6 . Rigidly foldable parallelogram twist configurations for $\theta<\alpha_{\min }$.

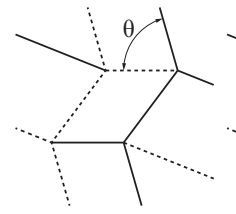

(a) BABA

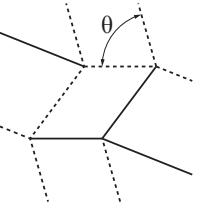

(b) BBAA

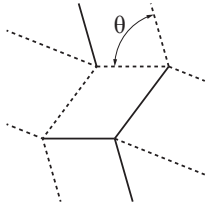

(c) BAAB

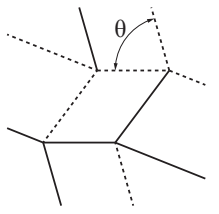

(d) $\mathrm{ABAB}$

FIGURE 7. Rigidly foldable parallelogram twist configurations for $\alpha_{\min }<\theta<90^{\circ}$.

(7) and/or (8). The multipliers for the other two vertices are found by substituting into Equations (7) and (8), resulting in

$$
\begin{gathered}
\mu_{A}=\frac{\cos \left(\frac{1}{2}(\alpha+\theta)\right)}{\cos \left(\frac{1}{2}(\alpha-\theta)\right)} \\
\mu_{B}=-\frac{\sin \left(\frac{1}{2}(\alpha+\theta)\right)}{\sin \left(\frac{1}{2}(\alpha-\theta)\right)}
\end{gathered}
$$

The product of any two of these Type-A multipliers and two of these Type$\mathrm{B}$ vertices is equal to one, satisfying Equation (6). Therefore, a parallelogram, rhombus, or isosceles trapezoid twist is rigidly foldable if it contains two Type-A and two Type-B vertices. The exception is where the twist angle is equal to the interior angle of a Type-A vertex, where infinite multipliers are obtained. Conversely, twists with unequal numbers of Type-A and Type-B vertices cannot satisfy Equation (6) and are not rigidly foldable.

Figure 6 shows the four rigidly foldable configurations for a parallelogram twist where the twist angle $(\theta)$ is smaller than any of the interior angles (again allowing for cyclic permutation and/or global parity reversal). Figure 7 shows the four rigidly foldable configurations for the case where one of the sets of interior angles is less than $\theta$. Figure 8 shows the six rigidly foldable configurations for an isosceles trapezoid for the case where the twist angle is less than any of the interior angles. Figure 9 shows the six rigidly foldable configurations for the case where the twist angle is greater than one pair of interior angles.

\subsection{Scalene Trapezoid Twists.}




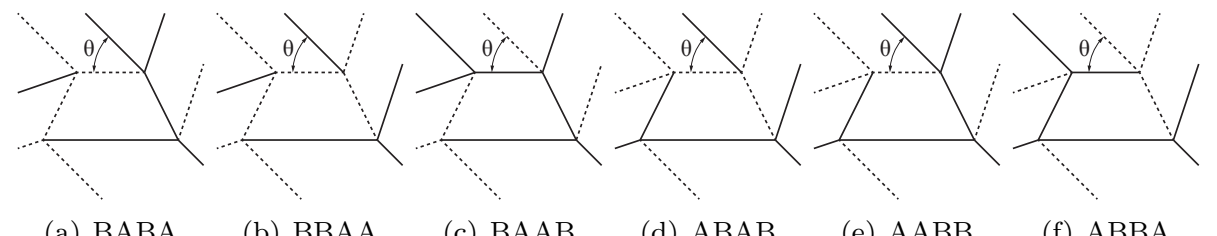
(a) BABA
(b) BBAA
(c) BAAB
(d) $\mathrm{ABAB}$
(e) $\mathrm{AABB}$
(f) $\mathrm{ABBA}$

FiguRE 8. Rigidly foldable isosceles trapezoidal twist configurations for $\theta<\alpha_{\min }$.

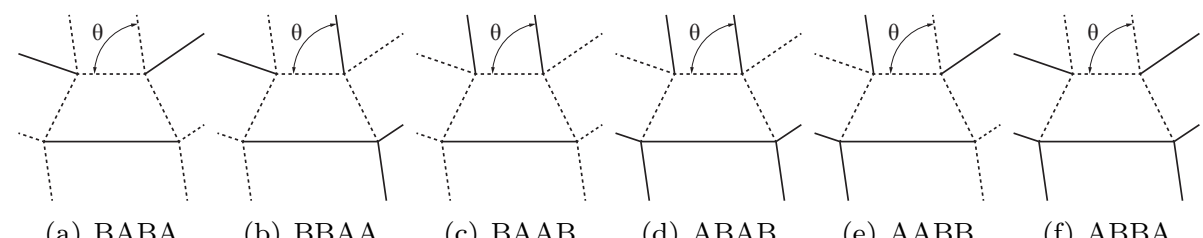
(a) BABA
(b) BBAA
(c) $\mathrm{BAAB}$
(d) $\mathrm{ABAB}$
(e) $\mathrm{AABB}$
(f) ABBA

Figure 9. Rigidly foldable isosceles trapezoidal twist configurations for $\alpha_{\min }<\theta<90^{\circ}$.

Theorem 5.3 (Scalene Trapezoid Twists). A scalene trapezoid twist is rigidly foldable if and only if the pairs of supplementary interior angles each include a Type-A and a Type- $B$ vertex and the twist angle is not equal to the interior angle of a Type-A vertex.

The proof is as follows. If $\alpha_{1}$ and $\alpha_{2}$ are two non-supplementary interior angles, then of necessity the other two interior angles must be the supplements to $\alpha_{1}$ and $\alpha_{2}$. As can be seen from Equations (7), (8), (14), and (15), the product of the multipliers for two supplementary-angled vertices of opposite type is -1. Therefore, the product of two such sets is equal to 1, satisfying Equation (6). Therefore, a scalene trapezoidal twist is rigidly foldable if the two sets of supplementary interior angles each have a Type-A and a Type-B vertex. Table 1 shows the possible vertex configurations for a rigidly foldable scalene trapezoid twist where Vertex 1 and Vertex 2 contain supplementary interior angles, as do Vertex 3 and Vertex 4 . The exception is if the twist angle is equal to the interior angle of a Type-A vertex, in which case infinite multipliers are obtained.

Theorem 3.1 rules out the possibility of a twist with all vertices of the same type. Any scalene trapezoid twist with 3 vertices of one type must include a set of supplementary vertices of opposite type. Since the product of the multipliers for such vertices is equal to -1 , the product of the multipliers of the other two vertices must also be -1 for it to be rigidly foldable. This dismisses the possibility of a scalene trapezoid twist with three vertices of the same type. The only remaining possibility is that one set of supplementary vertices of one type, and the other set is of the opposite type. However, this configuration is only rigidly foldable if $\alpha_{1}=\alpha_{2}$, resulting in an isosceles trapezoid or a parallelogram. Therefore, no conditions other than those stated in the previous paragraph result in a rigidly foldable scalene trapezoid twist. 
5.4. Kite Twists. The previous discussions apply for kites that are squares, rectangles, parallelograms, rhombuses, or trapezoids and the rigidly foldable configurations having two Type-A and two Type-B vertices. However, unlike the parallelogram, rectangle, or trapezoid twists, a kite twist can be rigidly foldable with three vertices of one type and one vertex of the other type.

We will call $\alpha$ and $\beta$ the two unique interior angles of a kite and $\theta$ the twist angle. For any combination of $\alpha$ and $\beta$ where $\alpha \neq \beta$ and $\alpha+\beta \neq \pi$ there exists a unique $\theta$ which results in a rigidly foldable kite twist for each of the six configurations with 3 vertices of one type and 1 of the other. The type labeling in this section labels the $\alpha$ vertex first and the $\beta$ vertex third, with the other two vertices second and fourth.

For the configuration with Type-A $\alpha$ and $\beta$ vertices and one other vertex of each type (AAAB/ABAA), a kite twist is rigidly foldable if

$$
\cos (\theta)=\frac{\sin (\alpha+\beta)}{\sin (\alpha)+\sin (\beta)}
$$

For the configuration with Type-B $\alpha$ and $\beta$ vertices and one other vertex of each type (BABB/BBBA), a kite twist is rigidly foldable if

$$
\cos (\theta)=-\frac{\sin (\alpha+\beta)}{\sin (\alpha)+\sin (\beta)}
$$

For the configuration with Type-A $\alpha$ vertex, Type-B $\beta$ vertex, and two other Type-A vertices (AABA), a kite twist is rigidly foldable if

$$
\cos ^{2}\left(\frac{\theta}{2}\right)=\frac{4 \cos \left(\frac{\alpha}{2}\right) \sin \left(\frac{\beta}{2}\right) \sin ^{2}\left(\frac{\alpha+\beta}{4}\right)}{\sin (\alpha)-\sin (\beta)+2 \sin \left(\frac{\alpha+\beta}{2}\right)}
$$

For the configuration with Type-A $\alpha$ vertex, Type-B $\beta$ vertex, and two other Type- $\mathrm{B}$ vertices $(\mathrm{ABBB})$, a kite twist is rigidly foldable if

$$
\cos ^{2}\left(\frac{\theta}{2}\right)=\frac{4 \cos \left(\frac{\alpha}{2}\right) \sin \left(\frac{\beta}{2}\right) \cos ^{2}\left(\frac{\alpha+\beta}{4}\right)}{\sin (\beta)-\sin (\alpha)+2 \sin \left(\frac{\alpha+\beta}{2}\right)}
$$

For the configuration with Type-B $\alpha$ vertex, Type-A $\beta$ vertex, and two other Type-A vertices (BAAA), a kite twist is rigidly foldable if

$$
\cos ^{2}\left(\frac{\theta}{2}\right)=\frac{4 \sin \left(\frac{\alpha}{2}\right) \cos \left(\frac{\beta}{2}\right) \sin ^{2}\left(\frac{\alpha+\beta}{4}\right)}{\sin (\beta)-\sin (\alpha)+2 \sin \left(\frac{\alpha+\beta}{2}\right)}
$$

Table 1. Rigidly Foldable Scalene Trapezoid Twist Vertex Types

\begin{tabular}{c|cccc}
\hline \hline Case & Vertex 1 & Vertex 2 & Vertex 3 & Vertex 4 \\
\hline 1 & $\mathrm{~B}$ & $\mathrm{~A}$ & $\mathrm{~B}$ & $\mathrm{~A}$ \\
2 & $\mathrm{~B}$ & $\mathrm{~A}$ & $\mathrm{~A}$ & $\mathrm{~B}$ \\
3 & $\mathrm{~A}$ & $\mathrm{~B}$ & $\mathrm{~B}$ & $\mathrm{~A}$ \\
4 & $\mathrm{~A}$ & $\mathrm{~B}$ & $\mathrm{~A}$ & $\mathrm{~B}$ \\
\hline
\end{tabular}


10THOMAS A. EVANS, ROBERT J. LANG, SPENCER P. MAGLEBY, AND LARRY L. HOWELL

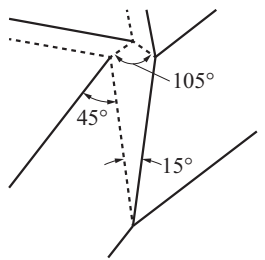

(a) ABAA

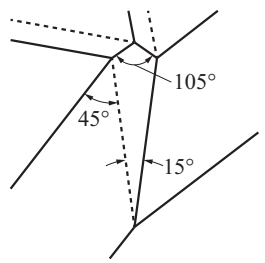

(b) $\mathrm{AAAB}$

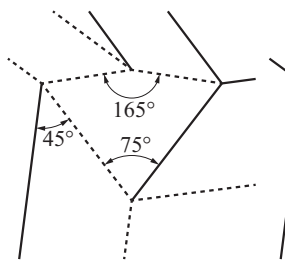

(c) BBBA

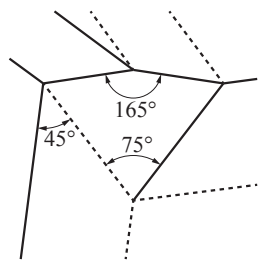

(d) BABB

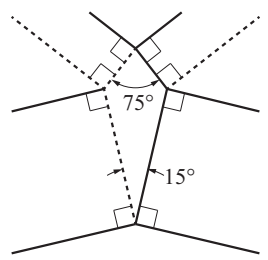

(e) $\mathrm{AABA} / \mathrm{BBAB}$

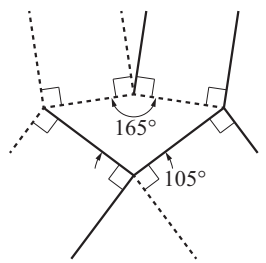

(f) $\mathrm{ABBB} / \mathrm{BAAA}$

Figure 10. Rigidly Foldable Kite Twists With Integer Angles. The $\alpha$ vertex is shown on the bottom in these figures $\left(\alpha=15^{\circ}\right.$ in (a)) and the $\beta$ vertex is shown on top $\left(\beta=105^{\circ}\right.$ in (a)). The alphabetical labeling lists the $\alpha$ vertex first and then moves clockwise around the central polygon. Note that cases (e) and (f) may be rotated and/or mirrored to obtain any of the four configurations listed.

For the configuration with Type-B $\alpha$ vertex, Type-A $\beta$ vertex, and two other Type- $\mathrm{B}$ vertices $(\mathrm{BBAB})$, a kite twist is rigidly foldable if

$$
\cos ^{2}\left(\frac{\theta}{2}\right)=\frac{4 \sin \left(\frac{\alpha}{2}\right) \cos \left(\frac{\beta}{2}\right) \cos ^{2}\left(\frac{\alpha+\beta}{4}\right)}{\sin (\alpha)-\sin (\beta)+2 \sin \left(\frac{\alpha+\beta}{2}\right)}
$$

Figure 10 shows the twists which result from the integer angle solutions to Equations (16) to (21).

\section{Regular Polygon Twists}

For an $n$-sided regular polygon, we define the interior angle at each vertex as $\alpha$, where

$$
\alpha=\pi-\frac{2 \pi}{n}
$$

If an $n$-degree regular polygon twist has $a$ Type-A vertices and $b$ Type-B vertices, then from Equation (6)

$$
\mu_{A}^{a} \times \mu_{B}^{b}=1
$$

where of necessity

$$
a+b=n
$$

Substituting Equations (7) and (8) into Equation (23) and simplifying gives 


$$
\left(\frac{\sin \left(\frac{1}{2}(\alpha-\theta)\right)}{\sin \left(\frac{1}{2}(\alpha+\theta)\right)}\right)^{a}\left(\frac{-\cos \left(\frac{1}{2}(\alpha-\theta)\right)}{\cos \left(\frac{1}{2}(\alpha+\theta)\right)}\right)^{b}=1
$$

Substituting Equation (22) into Equation (25) yields

$$
\left(\frac{\cos \left(\frac{\pi}{n}+\frac{\theta}{2}\right)}{\cos \left(\frac{\pi}{n}-\frac{\theta}{2}\right)}\right)^{a}\left(-\frac{\sin \left(\frac{\pi}{n}+\frac{\theta}{2}\right)}{\sin \left(\frac{\pi}{n}-\frac{\theta}{2}\right)}\right)^{b}=1
$$

This results in the following theorem:

Theorem 6.1 (Rigidly Foldable Regular Polygon Twists). A regular, n-degree polygon twist with a Type- $A$ vertices, $b$ Type- $B$ vertices, and twist angle $\theta$ is rigidly foldable if and only if Equation (26) is satisfied.

$g$

From Equations (9) and (23) it can be seen that there exist no rigidly foldable regular polygon twists where all vertices are of the same type. However, for each unique $a, b$ and $n$, with $0<a<n$ and $n>4$, there exists one unique twist angle

TABle 2. Twist Angles for Rigidly Foldable Regular Polygon Twists

\begin{tabular}{ccccc}
\hline \hline $\begin{array}{c}\text { Degree } \\
\mathrm{n}\end{array}$ & $\begin{array}{c}\text { Type-A } \\
\mathrm{a}\end{array}$ & $\begin{array}{c}\text { Type-B } \\
\mathrm{b}\end{array}$ & $\begin{array}{c}\text { Twist Angle } \\
\theta\left({ }^{\circ}\right)\end{array}$ & $\begin{array}{c}\text { Inner Angle } \\
\beta\left(^{\circ}\right)\end{array}$ \\
\hline 4 & 2 & 2 & $\neq 90.0$ & 90.0 \\
5 & 1 & 4 & 107.1 & 108.0 \\
5 & 2 & 3 & 96.8 & 108.0 \\
5 & 3 & 2 & 83.2 & 108.0 \\
5 & 4 & 1 & 72.9 & 108.0 \\
6 & 1 & 5 & 117.4 & 120.0 \\
6 & 2 & 4 & 104.5 & 120.0 \\
6 & 3 & 3 & 90.0 & 120.0 \\
6 & 4 & 2 & 75.5 & 120.0 \\
6 & 5 & 1 & 62.6 & 120.0 \\
7 & 1 & 6 & 124.1 & 128.6 \\
7 & 2 & 5 & 110.7 & 128.6 \\
7 & 3 & 4 & 96.8 & 128.6 \\
7 & 4 & 3 & 83.2 & 128.6 \\
7 & 5 & 2 & 69.3 & 128.6 \\
7 & 6 & 1 & 55.9 & 128.6 \\
8 & 1 & 7 & 129.6 & 135.0 \\
8 & 2 & 6 & 115.9 & 135.0 \\
8 & 3 & 5 & 102.7 & 135.0 \\
8 & 4 & 4 & 90.0 & 135.0 \\
8 & 5 & 3 & 77.3 & 135.0 \\
8 & 6 & 2 & 64.1 & 135.0 \\
8 & 7 & 1 & 50.4 & 135.0 \\
\hline
\end{tabular}


which satisfies Equation (26). This gives us the following result for regular polygon twists:

Theorem 6.2 (Rigidly Foldable Regular Polygon Twist Angles). For an n-degree regular polygon twist with $n>4$, there exist $n-1$ unique twist angles which result in a rigidly foldable twist.

Table 2 shows the twist angles which result in a rigidly foldable twist for regular pentagon, hexagon, heptagon, and octagon twists. For any even polygon twist, a $90^{\circ}$ twist angle is rigidly foldable with $a=n / 2$ and $b=n / 2$. It can be seen that the twist angles larger than $90^{\circ}$ are complementary to the twist angles for the opposite configuration. For $n>4$ rigidly foldable regular polygon twists, all interior folds must have the same parity because the interior angle is the largest angle at each vertex. For a square twist with $a=b=2$, Equation (26) is true for any value of $\theta$ other than $90^{\circ}$, where it becomes undefined.

\section{Conclusions}

We described a method for evaluating the rigid foldability of origami tessellations. We then applied this method to origami twists to discover what parameters allow an origami twist to be rigidly foldable. It was shown that there is no possible configuration for a rigidly foldable triangle twist. It was also shown that many possible rigidly foldable quadrilateral twists exist. Finally, a method for determining twist angles for a rigidly foldable regular polygon twist was presented. This method was used to calculate all possible twist angles for rigidly foldable regular polygons of degree eight or less.

\section{Acknowledgments}

This paper is based on work supported by the National Science Foundation and the Air Force Office of Scientific Research through NSF Grant No. EFRI-ODISSEI1240417.

\section{REFERENCES}

[Francis et al. 13] K. C. Francis, J. E. Blanch, S. P. Magleby, and L. L. Howell. "Origami-like creases in sheet materials for compliant mechanism design." Mechanical Sciences 4 (2013), $371-380$.

[Huffman 76] David A. Huffman. "Curvature and Creases: A Primer on Paper." IEEE Transactions on Computers C-25 (1976), 1010 - 1019.

[Hull 03] Thomas Hull. "Counting Mountain-Valley Assignments for Flat Folds." Ars Combinatoria 67.

[Kuribayashi et al. 06] Kaori Kuribayashi, Koichi Tsuchiya, Zhong You, Dacian Tomus, Minoru Umemoto, Kahiro Ito, and Masahiro Sasaki. "Self-deployable origami stent grafts as a biomedical application of Ni-rich TiNi shape memory alloy foil." Materials Science and Engineering A 419 (2006), 131 - 137.

[Lebee and Sab 10] A. Lebee and K. Sab. "Transverse shear stiffness of a chevron folded core used in sandwich construction." International Journal of Solids and Structures 47 (2010), 2620 2629 .

[Miura 85] Koryo Miura. "Method of Packaging and Deployment of Large Membranes in Space." Intstitute of Space and Asronautical Science 618 (1985), 1 - 9.

[Pickett 07] G. T. Pickett. "Self-folding origami membranes." EPL 78.

[Tachi 10] Tomohiro Tachi. "Freeform Rigid-Foldable Structure using Bidirectional Flat-Foldable planar Quadrilateral Mesh." In Advances in Architectural Geometry 2010. Springer Vienna, 2010 . 
[Tachi 11] Tomohiro Tachi. "Rigid-Foldable Thick Origami." In Origami 5, 2011.

[Zirbel et al. 13] Shannon A. Zirbel, Robert J. Lang, Mark W. Thomson, Deborah A. Sigel, Phillip E. Walkemeyer, Brian P. Trease, Spencer P. Magleby, and Larry L. Howell. "Accommodating Thickness in Origami-Based Deployable Arrays." Journal of Mechanical Design 135.

Department of Mechanical Engineering, Brigham Young University, Provo, UT 84602, U.S.A.

E-mail address: tevans11@byu.edu

Lang Origami, Alamo, CA 94507, U.S.A.

E-mail address: robert@langorigami.com

Department of Mechanical Engineering, Brigham Young University, Provo, Ut 84602, U.S.A.

E-mail address: magleby@byu.edu

Department of Mechanical Engineering, Brigham Young University, Provo, Ut 84602, U.S.A.

E-mail address: 1howell@byu.edu 